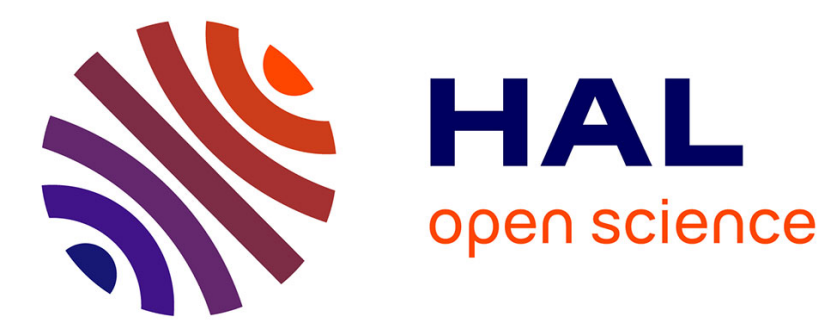

\title{
Maître ou Esclave? Benito Cereno ou les chausse-trappes d'un récit truqué
}

Serge Meitinger

\section{To cite this version:}

Serge Meitinger. Maître ou Esclave? Benito Cereno ou les chausse-trappes d'un récit truqué. Alizés: Revue angliciste de La Réunion, 1992, Pouvoirs et programme du CAPES, 02-03, pp.33-46. hal02338446

\section{HAL Id: hal-02338446 \\ https://hal.univ-reunion.fr/hal-02338446}

Submitted on 30 Oct 2019

HAL is a multi-disciplinary open access archive for the deposit and dissemination of scientific research documents, whether they are published or not. The documents may come from teaching and research institutions in France or abroad, or from public or private research centers.
L'archive ouverte pluridisciplinaire HAL, est destinée au dépôt et à la diffusion de documents scientifiques de niveau recherche, publiés ou non, émanant des établissements d'enseignement et de recherche français ou étrangers, des laboratoires publics ou privés. 


\title{
Maître ou esclave ? \\ Benito Cereno \\ ou les chausse-trappes d'un récit truqué
}

\author{
Serge Meitinger
}

Université de La Réunion

Le Pouvoir est un noeud tout comme le Récit. Si l'on ne sait en déchiffrer le lacs, il faut le trancher mais le mystère demeure, inentamé. Ce n'est pas par hasard qu'au coeur même du texte de Melville intitulé Benito Cereno un vieux marin tend au capitaine Delano un noeud complexe - composite - avec l'injonction impérieuse bien que furtive : "Débrouillez, tranchez, vite" (246)1. Ce noeud et l'invite qui l'accompagne sont l'emblème de la situation où se trouve engagé le lecteur du récit tel qu'il est agencé par Melville : dans les deux cas, il s'agit de souligner la nécessité d'une interprétation et d'une décision appropriées, liées à un redoutable, à un agaçant enjeu qui est le jeu $d u$ sens; dans les deux cas, il y a tromperie et il y va de la sécurité - de la sûreté - de l'interprète car le Récit (celui que propose Don Benito Cereno, celui qu'aménage le narrateur, celui que noue le Pouvoir à sa propre image) est "un piège" (selon l'expression de Louis Marin)2

\section{Les signes et les modèles}

Qui aborde un corps social tout autre que celui dans lequel il est né éprouve un vif sentiment d'étrangeté qui peut confiner au malaise voire à l'angoisse. En effet, le nouvel arrivant repère d'emblée ce qui lui apparait comme des violences, des dissonances ou des inconvenances, il remarque les inégalités, les faiblesses, les injustices patentes et il s'étonne que tout cela puisse tenir ainsi. Il voit comme à nu fonctionner les rouages d'une machinerie complexe dont le principe lui échappe : il sait qu'il y a des rapports d'autorité

1. Nous citons le texte de Melville dans la traduction française de Pierre Leyris : Benito Cereno et autres Contes de la Véranda, Coll. L'Imaginaire, Paris: Gallimard, 1977, pp. 193-317.

2. Nous empruntons l'expression au titre du livre de Louis Marin : Le Récit est un piege, Coll. Critique, Paris : Editions de Minuit, 1978 ; mais nous n'exploitons en rien la problématique adoptée par cet auteur. 
à l'oeuvre dans la dynamique qu'il envisage mais il sait aussi que ces derniers ne sont jamais de purs rapports de force et c'est la table des valeurs qui lui fait défaut.

Nous sommes en 1799 et le capitaine Amasa Delano, de Duxbury, Massachusetts, commandant un phoquier de fort tonnage, est, peu d'années après l'Indépendance, le type même de l'Américain attaché à l'égalité, à la démocratie et au respect de la personne. Ces postulations idéologiques et morales s'allient en lui à une évidente bonhomie et à une foi solide en la justice de la Providence divine : l'homme a a priori peu de sympathie pour un système fondé sur l'esclavage, mais il s'efforce d'apprécier la diversité des cas et des tempéraments humains avant que de se prononcer. Le navire espagnol délabré, commandé par Don Benito Cereno, ne peut lui apparaître que comme le vestige d'un monde féodal se parant encore des débris d'une splendeur déchue mais il tente, pour l'accepter, d'en comprendre les données. Sa première impression est d'irréalité foncière : le bateau étranger lui apparaît sur le mode d'un vaisseau fantôme, peuplé de "silhouettes sombres et mouvantes pareilles à des Frères Noirs arpentant leurs cloîtres" (198). L'effet d'étrangeté est accru par le contexte : le narrateur oppose sur ce point l'entrée "dans une maison étrangère" à l'approche d'un bateau inconnu :

La maison comme le navire, l'une de ses murs et de ses volets, l'autre de ses hauts pavois semblables à des remparts, jusqu'au dernier moment dérobent au regard leur organisation intérieure ; mais le cas du navire offre en outre cette particularité : le spectacle vivant qu'il recèle, à l'instant soudain qu'il est révélé, produit en quelque sorte, par contraste avec l'océan vide qui l'environne, l'effet d'un enchantement. Le navire paraît irréel ; ces costumes, ces gestes et ces visages étrangers semblent n'être qu'un mirage fantomatique surgi des profondeurs qui reprendront bientôt ce qu'elles ont livré. (201)

Et cette sensation qui laisse affleurer la présence occulte et opaque d'un monde englouti, d'un secret sans doute terrible, ne s'estompera jamais bien que le capitaine, en marin averti, s'efforce avant tout de se faire l'herméneute des signes concrets et patents qu'il peut appréhender. Il remarque ainsi d'emblée que le pilote du vaisseau espagnol ignore manifestement la configuration du havre où il s'apprête à mouiller (une petite île désertique, située à l'extrémité méridionale de la côte du Chili) et il s'intéresse très vite surtout aux curieuses relations d'autorité qui règlent la vie à bord. La société qui se manifeste à ses yeux lui apparaît comme une sorte de monde à l'envers où les Noirs, censés être les esclaves et malgré une apparente soumission, dominent en fait les Blancs, ne serait-ce d'abord que par le nombre. Comme des figures tutélaires, placées au-dessus de la cohue et vaguement menaçantes, se tiennent "quatre nègres grisonnants" (202) chargés tout en faisant de l'étoupe de veiller à la discipline de la foule qu'ils rappellent périodiquement à l'ordre. Pourtant 
de multiples actes d'insubordination demeurent impunis et cet étonnant laxisme suscite une légitime curiosité envers la personnalité de Don Benito Cereno, seul maître après Dieu du navire espagnol, autour de qui va se cristalliser toute l'attention du capitaine Delano. Cereno est dans un état de délabrement physique et moral bien en rapport avec l'état de son bateau et la faiblesse qui est la sienne rend plus touchant le dévouement de Babo, son serviteur noir, et plus mystérieuse son emprise de chef sur l'ensemble de l'équipage. Etre taciturne et inconstant, comme dégénéré par rapport à l'aristocratie dont il est issu (surtout aux yeux d'un démocrate comme Delano), Don Benito Cereno ne cesse de troubler le jugement par son attitude ambivalente ei ses constants revirements d'humeur : sa personne et tout son comportement constituent le noeud de l'intrigue parce qu'ils recèlent à la fois l'énigme du Pouvoir et la puissance propre au Récit. C'est en effet Cereno qui raconte l'aventure extraordinaire du San Dominik, parti de Buenos-Aires à destination de Lima avec un chargement varié dont un lot d'esclaves et pris au large du Cap Horn dans de violentes tempêtes qui décimèrent l'équipage espagnol et démantelèrent les structures du voilier. Des épidémies succédèrent aux intempéries et des bonaces : comme enfermé en un terrible Pot au Noir, le vaisseau égaré erra sur place et épuisa ses ressources en ce vain périple. Ce récit dont Delano ne met d'abord pas en doute la véracité lui laisse toutefois supposer l'incompétence d'un Cereno privé de ses officiers et de ses marins les plus aguerris. Et, dans l'esprit de Delano, de l'incompétence du commandant à la dégradation de son autorité et de la discipline en général il n'y a qu'un pas : d'où le laxisme constaté. Mais comment expliquer alors l'espèce de despotisme, détourné et parfois presque sadique, qui par ailleurs semble être le propre de Cereno ? Le cérémonial qu'il impose au géant noir Atufal trahit une conception particulièrement complexe du respect, de la force et de la dignité : ce dernier, dûment enchaîné, comparaît toutes les deux heures devant le commandant (222-3) et s'entend demander un aveu de soumission qu'il se refuse obstinément à faire, et ce depuis soixante jours : l'esclave qui fut noble en sa nation oppose ainsi, au dire de Delano, "un esprit royal" (223) à la contrainte servile qui tente de l'humilier, mais le démocrate ne comprend pas pourquoi ce conflit d'aristocrates ne débouche pas sur un pardon magnanime. En effet Cereno ne tient pas à user de torture et le rebelle n'est tel que parce qu'il ne veut pas prononcer la formule qui consacrerait sa déchéance ; par ailleurs, son comportement resté soumis. Le modèle aristocratique et chevaleresque, suggéré ici par la situation et par les caractères, ne rend pas bien compte des signes émis par les personnages en acte. De même un geste de Cereno va jeter le doute sur la nature des rapports établis entre Babo et son maître : le serviteur déploie à l'égard du malade, épuisé nerveusement, une sollicitude de tous les instants qui prend l'allure d'une profonde affection. Or, Babo l'ayant blessé d'un mouvement malencontreux de son rasoir, Cereno se venge en iui balafrant la joue d'un coup du même rasoir. L'incident détruit un instant dans l'esprit de l'Américain l'image 
d'harmonie et d'humanité qui semblait être celle du couple maître-serviteur : il y voit d'abord une perversion due à l'esclavagisme mais il rétablit vite les choses en évoquant à part soi une péripétie du même ordre qu' "une querelle d'amoureux" (267). Cependant, une fois de plus, le modèle préétabli ne s'applique pas intégralement à l'analyse des faits et des signes et l'herméneute se trouve comme crucifié en un carrefour où se recroisent des données contradictoires induisant des interprétations divergentes :

D'abord, l'affaire au mousse espagnol assailli à coups de couteau par l'esclave ; et cela au vu de Don Benito. En second lieu, le noir Atufal tyranniquement traité par Don Benito, à la manière d'un taureau du Nil qu'un enfant mènerait par un anneau passé dans les narines. Troisièmement, le matelot piétiné par les deux nègres, insolence qui avait passé sans l'ombre d'une réprimande. Quatrièmement, la rampante soumission dont faisaient preuve à l'égard de leur maitre les eléments subalternes du navire, des noirs pour la plupart, comme s'ils craignaient de provoquer pour la moindre inadvertance son déplaisir despotique. (250)

Que l'on use de la métaphore du carrefour ou de celle du noeud, il est indéniable que le brave capitaine Delano est ici au coeur du dilemme et qu'il ne sait dénouer "l'enchevêtrement de ses pensées" semblable "à celui du chanvre" (246). Laxisme et/ou despotisme ? Quelles sont les voies tortueuses du Pouvoir de Cereno ? Le sentiment du danger ne peut que croître dans l'esprit de l'interprète car l'impression qui s'impose de façon à peine formulée est qu'une force latente opère dans l'ombre, tout autre qu'une pure et simple contrainte physique : une puissance de nature morale voire spirituelle. L'idée de trahison grandit et surgit à plusieurs reprises : Benito Cereno pourrait bien être l'âme damnée d'un complot visant à s'emparer du phoquier. Mais l'Américain se laisse rassurer par les faits qui ne cessent de révéler l'insigne faiblesse physique et morale de son hôte et par les marques de déférence que ce dernier lui prodigue. Et jusqu'au moment du renversement ou de la révélation, il doute et se tient dans l'expectative, croyant assez fermement en la justice de la Providence pour ne pas s'inquiéter plus qu'il ne convient à une âme chrétienne. C'est en fait Benito Cereno lui-même qui, en sautant du pont de son navire dans la baleinière de Delano, va trancher le dilemme : la vraie nature des relations de Pouvoir entre le maître et l'esclave apparaît au moment où Babo tente de poignarder Cereno. Le capitaine Delano est vivement "frappé" (286) par cette révélation et l'effet de cette surprise est souligné. Toutefois pour le lecteur qui a tenté de démêler les fils de l'intrigue dans le sillage du bienveillant interprète, le choc n'est pas moins grand. En effet peu de récits donnent à ce point au lecteur le sentiment rétrospectif d'avoir été floué et sans cesse manoeuvré : le moment de "l'éclair révélateur" qui illumine l'esprit de Delano éclaircit certes - et pour lui aussi - les données matérielles du mystère mais il rend en même temps nécessaire un retour réflexif sur la nature même du récit tel qu'il a été conduit jusque-là. Le choc éprouvé par le lecteur 
a ainsi une vertu herméneutique. De fait la fin du texte rapportant la déposition de Cèreno devant le tribunal qui juge les mutins n'élucide pas vraiment, malgré une apparente symétrie, l'énigme déployée par la première et la plus longue partie du récit : ce qui a été lu et donc, à sa façon, vécu continue à peser et reste à interpréter car l'étonnante maîtrise du narrateur qui, pour notre plus grande confusion, a veillé à multiplier et à mêler les signes comme les modèles et nous a ainsi pris au piège du Récit, ne nous en délivre pas.

\section{Pouvoirs du narrateur}

L'art suprême du conteur et son pouvoir propre sont de faire de chaque indice qu'il présente ou analyse un signe qui concourt au sens du tout : chez un écrivain digne de ce nom rien n'est insignifiant ni hasardé. Mais l'instance narrative mise en jeu peut être le moyen d'une ruse et interdire momentanément la pleine appréhension de ce qui est avancé et en même temps ảissimulé. Le narrateur peut ainsi, sciemment, égarer son lecteur en le détournant de la réalité des faits. De la sorte toutes les amorces nécessaires à une juste et précise compréhension sont déjà là mais le lecteur est condamné à ne saisir le jeu du sens en sa plénitude qu'après coup. Une relecture s'avère nécessaire et certains textes (comme celui-ci) sont peut-être écrits pour être relus tout autant que pour être lus : ce sont eux qui posent avec le plus d'acuité le problème de la vérité narrative dans ses rapports avec le Pouvoir et avec le Récit.

Le récit que développe cette grosse nouvelle ou ce petit roman est mené le plus souvent selon la technique narrative dite du point de vue : nous voyons avec les yeux du capitaine Delano et nous lisons dans ses pensées. Mais ce parti-pris n'exclut pas les interventions d'un narrateur omniscient qui brosse le décor et qui glisse presque subrepticement des indications ou des jugements nourrissant le récit d'informations qui font défaut au personnage-herméneute. La narration en apparence linéaire se dédouble et doit se lire sur deux plans à la fois, le second plan n'apparaissant toutefois en pleine lumière qu'à la relecture, une fois la révélation acquise. Le jour qui se lève à l'orée du texte a quelque chose de gris et voile (et il restera tel), quelque chose de louche aussi comme les faits qui seront narrés ; le lieu où se situe la rencontre des deux vaisseaux est un endroit désertique et il est dit un "lieu sans lois" : le décor est en osmose avec ce qui va être montré, il contribue à l'atmosphère morale. Mais cette analyse que le nariateur nous incite à faire avec lui échappe en partie à l'entendement du capitaine Delano dont les capacités de jugement sont ainsi mises en cause :

A considérer la solitude de ce lieu sans lois et la sorte d'histoires que l'on associait alors à ces mers, la surprise du capitaine Delano eût peut-Etre augmenté jusqu'au malaise si notre homme n'avait été d'un naturel singulièrement bon et 
confiant, peu enclin à prendre l'alarme - sauf après des incitations extraordinaires et répétées - lorsque ladite alarme impliquait une accusation de malignité contre son prochain. Quant à savoir, étant donné ce dont l'humanité est capable, si pareil trait révèle, outre un coeur bienveillant, une perception intellectuelle particulièrement rapide et pénétrante, nous abandonnerons cela au jugement des sages. (196)

Nous sommes prévenus : une âme naïve et généreuse qui n'a pas l'imagination du mal n'est sans doute pas l'interprète idéal pour une histoire dont la gravité transparaît déjà. Néanmoins, c'est à ce coeur confiant que l'auteur réserve le souci de l'enquête et il s'agit bien de chercher avec lui la vérité. Du point de vue de l'instance narrative cette quête progresse de deux manières en même temps : d'une part celui qui raconte se fait la voix du destin qui sait $d^{\prime}$ avance, d'autre part il suit et mime les méandres où ne cesse de se perdre la pensée du capitaine Delano. Le narrateur laisse presque le lecteur s'égarer avec le brave Américain, car s'il glisse dans son récit quelques indices qui par ailleurs échappent au capitaine il n'en dit pas assez pour dévoiler le fin mot de l'énigme et il s'arrange pour revoiler aussitôt ce qu'il vient de découvrir, bien plus il monte de toutes pièces une anecdote décisive qui peut passer pour un trucage majeur.

La voie du destin, présente tout au long du texte, use de ce que l'on appelle dans un tout autre contexte l'ironie tragique. La description de la proue du vaisseau espagnol en est un bel exemple : après avoir montré que cette partie du navire, apparemment délabrée ou en réfection, est voilée d'une toile et signale la devise qu'elle porte : "Suivez votre chef", le narrateur termine son paragraphe sur l'évocation "d'un roulement de corbillard" (200). De même, un peu plus loin, devant Cereno, Delano va regretter ouvertement que les restes d'Alexandro Aranda, le propriétaire des esclaves et l'ami du commandant espagnol, ne soient plus à bord : cette macabre hypothèse et l'image très vive qu'elle induit provoquent l'évanouissement de Cereno (220-1). Juste après le coup de théâtre provoqué par le saut de Cereno dans la baleinière suivi de la tentative de Babo et juste avant l'assaut, "le linceul de toile" qui enveloppe la proue sera arraché et le squelette d'Aranda qu'il cachait va apparaître en pleine lumière : seconde révélation confirmant la première et permettant enfin de comprendre les allusions restées opaques (286-7). Ainsi les indices semés par le narrateur usant pleinement de sa faculté d'omniscience ne s'éclairent qu'après coup et ne trouvent toute leur saveur qu'à la relecture. L'on s'aperçoit ainsi que ce n'est pas par hasard ou pour le goût qu'il aurait des couleurs vives que Babo, se faisant barbier (260-2), fronce sous le menton de son maître en guise de simple serviette l'étendard d'Espagne ! L'irritation de Delano qui ne parvient pas à se trouver un instant seul avec Cereno malgré l'effort qu'il vient de faire pour le retrouver avant que Babo ne puisse les rejoindre revêt une formulation pour le moins ambiguë : "Que Dieu confonde ce fidèle serviteur, pensa le capitaine Delano ; quelle agaçante cö̈ncidence !" (276) De même le 
compliment ironique qu'il fait, juste après, à Cereno et la façon dont il interprète la réaction de ce dernier révèlent la naïveté de l'Américain :

- [...] "Ah ! Don Benito," ajouta-t-il en souriant, "malgré toute la licence que vous autorisez à certains égards, je crains qu'au fond vous ne soyez un maitre impitoyable."

De nouveau Don Benito sursauta ; et cette fois, pensa le bon marin, sous l'effet d'un vrai remords de conscience. (277)

Le narrateur se donne ainsi beau jeu : lui qui sait d'a vance peut faire ironiquement éclater (bien qu'a posteriori, en un temps second) la candeur de son personnage. Pour lui il ne saurait y avoir de "coïncidence" mais une implacable nécessité et, en la matière, il réalise une manière de petit chef d'oeuvre : au moment de l'adieu, Cereno qui a renoncé à voir Delano comprendre sa véritable situation s'exclame : "Allez, et que Dieu vous garde micux que moi, mon excellent ami" (283). Tout est dit et cependant rien ne passe : cette phrase est une sorte de modèle réduit de toute la première partie du récit dont elle reproduit l'ambivalence ; cinq minutes plus tard Cereno va sauter du pont de son propre navire.

Mais la prétendue ingénuitê de Delano n'est pas la seule cause d'un tel retard dans la compréhension : par des jugements orientés, par des mises en garde tendancieuses et sélectives le narrateur a en fait interdit au lecteur de progresser plus vite que le capitaine américain.Le champ ouvert aux soupçons du lecteur est certes plus large que celui que laboure consciencieusement Delano, mais son jugement est infléchi et ses perspectives limitées. C'est bien le narrateur et lui seul qui qualifie Benito Cereno de "demi-dément" (206) : l'analyse de l'Américain, il le signale, ne va pas encore jusque-là. Et ce jugement à l'emporte-pièce fait naître dans l'esprit de celui qui lit l'idée que le commandant espagnol est un irresponsable dont les propos et les actes n'ont pas forcément à offrir de sens logique. C'est toutefois dans sa façon de décrire la teneur et la qualité des rapports entre Cereno et son serviteur noir Babo que le machiavélisme du narrateur est le plus grand. Il cautionne d'emblée l'image idéale du serviteur attaché à la personne de son maître avec l'amour inquiet de l'animal familier et il réitérera à maintes reprises cette caution :

Auprès de lui se tenait un noir de faible stature, qui levait de temps à autre vers l'Espagnol, comme un chien de berger, un visage rude où se mêlaient également le chagrin et l'affection. (203)

D'ailleurs cette image persistera partiellement jusqu'au moment de l'adieu sur la coupée : Babo a servi de béquille à son maître ; compatissant, Delano a tendance à s'attarder mais "rencontrant le regard discrètement admonitoire du serviteur" (284), il précipite son départ. Toutefois des indices diver- 
gents se font également jour : les fréquentes interventions du serviteur dans la conversation que son maître entretient avec Delano irritent ce dernier. Elles visent à souligner la puissance du lien privilégié et comme absolu qui garantit la fidélité et la complicité de l'esclave ; elles ressemblent parfois à un rappel à l'ordre. Les rapports entre le maître et l'esclave révèlent en effet par éclairs leur ambivalence foncière : un propos de Delano, involontairement plein d'ironie tragique, suscite cette petite scène lourde de sous-entendus :

"Ah! Don Benito, vous semblez accorder à ce noir une grande place dans votre confiance ; en fait, c'est une manière de conseiller prive."

Sur quoi le serviteur leva les yeux avec un ricanement bonhomme, mais le maître tressaillit, comme sous l'atteinte d'une morsure venimeuse. Quelques instants s'écoulèrent avant que l'Espagnol se fût suffisamment ressaisi pour répondre ; ce qu'il fit enfin avec une froide contrainte, disant :

"Oui, Señor, j'ai confiance en Babo."

Ici Babo, changeant son ricanement de pure gaîté animale en un sourire intelligent, regarda son maître non sans gratitude. (230-1)

En fait, Delano, qui n'a pas apprécié les conciliabules chuchotés de Cereno et de son serviteur, qui est gêné par les questions indiscrètes posées sur son propre navire, qui a cru surprendre des signes de mise en garde à lui adressés par des matelots espagnols, soupçonne un complot dont la tête serait le commandant et Babo l'instrument : son ironie qui vise la fonction occulte de ce dernier est destinée à désarmer sa propre défiance. Cependant ce que nous laisse pressentir le narrateur à travers le comportement de Cereno est tout autre : $c^{\prime}$ est le maître qui subit la contrainte. La métaphore de l'animal familier s'est transmuée en celle d'animal venimeux. Mais pourquoi le narrateur évoque-t-il alors le "sourire intelligent" et la "gratitude" de l'esclave ? L'on attendrait plutôt un regard de triomphe ou de joie perverse. Est-ce une impression de Delano ? Le fait n' est pas donné pour tel. Est-ce une preuve de l'extraordinaire capacité de dissimulation propre à Babo ? L'indication est isolée et ne se complète de rien qui garantisse ce sens. Il semble qu'il s'agisse d'une résurgence ou d'une réhabilitation de l'image du serviteur fidèle qui vient d'être mis en cause. A notre avis, nous avons là un exemple de duplicité narrative : ces notations ne sont là que pour nous égarer, pour nous empêcher de prolonger notre étonnement et les soupçons que le récit vient d'induire en une réflexion construite et argumentée sur la nature exacte des rapports du maître et du serviteur. Toutefois le narrateur qui, par ailleurs, continue à propager le soupçon, nous réserve en la matière un trait plus marquant encore, destiné à enlever au lecteur la possibilité même de croire encore à la prééminence perverse de l'esclave sur le maître. L'attitude des deux protagonistes dont Delano ne cesse de questionner les propos et les gestes lui révèle une fois au moins (lors de la séance de rasage) et comme au naturel la vérité de leurs rapports : 
Maître ou esclave : Benito Cereno ou les chausse-trappes d'un récit truqué - 41

Toute la scène avait quelque chose de singulier, au moins pour le capitaine Delano qui, à considérer la posture des deux hommes, ne put chasser l'idée saugrenue qu'il voyait dans le noir un bourreau et dans le blanc un homme au billot. mais c'était là un de ces fantasmes capricieux qui apparaissent et s'évanouissent en un clin d'oeil et dont l'esprit le mieux réglé ne saurait sans doute se garder. (261-2)

La technique du point de vue est ici détournée en raison des prises de position successives du narrateur et ce pour dévaloriser formellement l'impression vivement ressentie par Delano et réduire la vérité qu'il vient d'entrevoir à un pur et simple fantasme. Mais l'image du bourreau est tenace : elle persiste dans l'esprit de Delano comme dans celui du lecteur et l'on s'attend à un égorgement. Il faudra d'ailleurs peu de temps pour voir couler le sang de Cereno : l'agitation que provoquent chez lui les propos de l'Américain se trouve liée à un malheureux coup de rasoir et le sang jaillit (263). Le narrateur insiste lourdement sur le caractère involontaire ou fortuit du geste : il s'agit de disculper Babo de toute velléité agressive. Mieux, quelques pages plus loin, c'est Cereno qui va devenir le bourreau et Babo l'innocente victime :

[Delano] se retourna et vit le nègre, portant sa main à sa joue. Le capitaine Delano s'avançá vers lui et s'aperçut que la joue saignait. Il était sur le point de lui en demander la cause, quand le soliloque plaintif du nègre l'instruisit :

"Ah ! Quand donc Maître sera-t-il guéri de sa maladie ? C'est la maladie qui rend méchant et qui le fait traiter Babo comme ça; couper Babo avec le rasoir parce que Babo, seulement par accident, a fait à Maître une seule petite égratignure, et ça pour le première fois depuis tant de jours! Ah! Ah! Ah ! (266-7)

Le narrateur a retourné l'image du bourreau et la "vision" de Delano peut bien être regardée comme un fantasme : le jugement à l'emporte-pièce qui faisait, dès le début, de Cereno un "demi-dément" semble trouver là sa pleine justification. Toutefois le narrateur ne montre pas le fait dont l'interprétation est entièrement laissée à Babo lui-même d'abord puis à Delano et ainsi il n'assume pas la responsabilité de l'événement bien qu'il en recueille tout le bénéfice narratif. De la sorte nous nous croyons en mesure de parler de mauvaise foi narrative : le seul but du narrateur nous semble avoir été de ménager avec une parfaite maîtrise et par tous les moyens dont il pouvait disposer l'effet majeur de son récit qui est le coup de théâtre ou le complet renversement provoqué par le geste désespéré de Cereno se jetant dans la baleinière. Il fallait que le lecteur fût aussi surpris que Delano lui-même et tout aussi gêné de n'a voir pas compris d'emblée alors que de multiples signes étaient prodigués en pure perte. 
Le geste de Cereno puis l'éclair qui illumine l'esprit de Delano marquent dans le récit et de façon absolue un avant et un après et ils relancent l'interprétation qu'ils radicalisent. Il faut lire et relire l'ensemble à leur sombre lumière. Car il ne s'agit pas là d'un dénouement au sens habituel : quelque chose reste noué, continue même à se nouer et va demeurer indémêlable. En effet la seconde partie du récit est plutôt frustrante bien qu'elle dévoile l'envers de la machination dont nous avons aussi été les victimes. Les faits s'éclairent et nous découvrons, comme dans un puzzle, les éléments qui nous manquaient pour s'ajuster aux signes déjà décelés et d'abord incompris ou mal interprétés mais cette recomposition à la fois sémiotique et herméneutique ne nous suffit pas : $c^{\prime}$ est le ressort ou la motivation centrale qui font défaut, c'est la persistance de l'horreur et de la peur qui ne s'explique pas. A partir de l'effrayante révélation qui nous bouleverse nous devons considérer en tant que lecteur et en tant qu'homme, au-delà des pouvoirs du narrateur, la vérité du Pouvoir lui-même en son essence noire.

\section{L'ombre portée}

La seconde partie du récit (de loin la plus ccurte) se donne pour l'envers ou le revers de la première et l'envers d'un monde à l'envers devrait être un monde à l'endroit. Pourtant, malgré un contre-récit circonstancié, rien ne retrouve vraiment son aplomb : le sentiment de l'irrémédiable s'impose. L'assaut donné au vaisseau des mutins par les hommes de Delano et qui devrait d'une certaine manière rétablir l'ordre commence par aggraver le gâchis : plusieurs des matelots espagnols, seuls survivants de l'équipage, sont tués par les Américains. Le second récit de Cereno, emprunté aux minutes du procès et destiné à corriger le premier récit, mensonger, a le défaut de ce genre de relations : une certaine sécheresse factuelle et le souci dominant de préciser les éventuelles responsabilités juridiques alors que l'essentiel pour nous se situe ailleurs. Le bateau était parti de Valparaiso et non de Buenos-Aires et le but des esclaves révoltés était de recouvrer leur liberté et une patrie : leur cruauté envers les Espagnols a revêtu constamment l'allure d'une vengeance personnelle et collective. Le tribunal de Lima tranche selon les lois en vigueur dans la vice-royauté et les principaux mutins seront condamnés à mort tandis que les biens restants (hommes et choses) seront partagés entre les ayants droit. Le tribunal a tranché, mais il n'a pas déchiffré l'étrangeté des faits qu'il a eu à connaître. En particulier, il ne saurait donner de suites à deux circonstances majeures qui s'imposent à lui mais qui, à ses yeux, rendent les événements difficiles à imaginer : la nature et la portée de l'influence de Babo sur les autres Noirs et surtout sur Cereno, l'étrange et tortueuse machination montée par les chefs des mutins (Babo et Atufal) pour s'emparer du phoquier et dont faillit être la victime le capitaine Delano en raison de sa trop grande générosité et de sa naïveté. Au moment où il lui faut conclure, le narrateur est conscient 
qu'il y a là comme une lacune et il se sent tenu de justifier les évidentes irrégularités de son récit :

Si la déposition de Benito Cereno a pu servir de clef pour forcer les rouages compliqués qui l'ont précédée, comme un caveau dont le vantail serait soudain écarté, la coque du San Dominik est à présent ouverte.

Jusqu'ici la nature du récit a non seulement rendu inévitables les enchevêtrements du début, mais encore exigé qu'un grand nombre de faits, au lieu d'être narrés dans l'ordre où ils s'étaient passés, fussent rétrospectivement ou irrégulièrement présentés ; $œ$ dernier cas est celui des passages suivants, qui concluront la relation. (313)

La métaphore de la clef chasse un instant celle du noeud mais c'est une clarté illusoire qui vient d'être répandue. Les "enchevêtrements" demeurent et le récit va s'achever comme il a commencé : sur une irrégularité. En fait le narrateur a noué son récit à l'image du pouvoir qui y est à l'oeuvre ou c'est l'essence même du Pouvoir qui a noué le Récit à son image. Après la reconquête de son vaisseau par les marins du phoquier, bien qu'apparemment "délivré de toute contrainte", Benito Cereno ne recouvre que temporairement" la santé et le libre exercice de sa volonté" (292). Il reste obsédé et marqué par ce qu'il a subi :

L'Espagnol répétait sans cesse combien il lui avait été pénible de jouer le rôle imposé par Babo. (313)

Au cours de ses conversations avec Delano, il ressasse les circonstances de leur rencontre et ne cesse de revivre la terrible journée qui représenta pour lui le paroxysme de son supplice. Il n'arrive pas à se reconnaître dans le Benito Cereno de ce jour-là qui conduisit si près de la mort - sans le vouloir mais sciemment - le généreux capitaine Delano, voué au pire des sorts en raison même de son âme charitable. Il n'admet pas non plus l'image de scélérat que s'est forgé de lui un court moment ce dernier. Quelqu'un qui s'est sur lui arrogé un pouvoir absolu a aliéné et détruit la personne de Don Benito Cereno parce qu'il l'a contraint à saccager lui-même en les dévoyant de l'intérieur toutes les valeurs qui représentaient pour lui la Loi et donnaient un sens à sa vie :

- "Vous êtes sauvé, Don Benito," s'écria le capitaine Delano, de plus en plus surpris et peiné ; "vous êtes sauvé ; qu'est-ce donc qui a jetê une telle ombre sur vous?"

- "Le nègre." (315-6)

L'ombre portée sur l'âme de Cereno est trop lourde pour lui et littéralement elle l'anéanti : même mort il devra encore soutenir le regard indompté de l'esclave lui-même mort. Mais l'ombre ici nommée et dévoilée nous révèle 
l'essence du Pouvoir. La loi du plus fort n'y limite jamais vraiment son emprise à une pure et simple confrontation physique qui soumettrait le plus faible. Le caractère maléfique du Pouvoir est que, ne pouvant faire fi des formes imposées par la Loi morale - c'est là son impératif catégorique ! -, il repose avant tout sur l'art de manipuler et d'imposer des signes, des images et des valeurs. Exercer le Pouvoir comme se soumettre au Pouvoir c'est, dans ses discours, ses décisions et ses actes, vivre sincèrement ou mimer de gré ou de force, consciemment ou non, un certain nombre de vertus, c'est faire allegeance formelle à des valeurs, à des normes et à des conventions qui se donnent pour des références stables. Le plus souvent celui qui exerce le Pouvoir tout comme celui qui s'y soumet adhèrent à l'idéologie qui légitime l'ordre établi dont le caractère tout relatif est ainsi gommé : le maître, propriétaire d'esclaves, ne doute pas de la supériorité de sa race qui lui donne le droit de s'assujettir une autre race, inférieure, et cette dernière ne devrait pas douter non plus de son infériorité donc de la légitimité de l'esclavage. Le maître ne se rend pas compte que non seulement il contraint l'esclave à lui être physiquement soumis mais encore qu'il exige de lui l'adhésion sincère ou feinte à son propre système de valeurs : la nature, la vérité, les vertus de l'esclave sont celles que lui reconnaît le maître. La révolte des esclaves renverse le rapport de force et aussi le sens des valeurs et il s'agit d'abord d'une vengeance. C'est la rencontre avec le phoquier qui va susciter une ruse liée aux enjeux fondamentaux du Pouvoir : il ne faut pas éveiller les soupçons d'où la nécessité de mimer des rapports d'autorité qui puissent passer pour normaux aux yeux des Américains. Cereno va être contraint, pour sauver sa vie et celle des Espagnols survivants, de jouer comme un rôle une fonction qu'il avait jusque-là considérée comme naturelle, il va devoir contrefaire ce qui pour lui a encore le plus de sens et de puissance morale : il se trouve désormais dans la situation spirituelle de l'esclave obligé de pratiquer un jeu qui l'aliène (mais c'est ici son propre jeu, retourné, démythifié, dévalué). La surveillance acharnée exercée par Babo, violence insidieuse de toutes les secondes, lui interdit tout écart, toute échappatoire : il est tenu de coller à un rôle dont l'inanité et la perversité intrinsèque deviennent les siennes. Et Babo détruit ainsi en lui toute espérance, d'abord en étouffant la vérité sous le masque mais surtout en utilisant celui-ci pour duper la générosité de Delano. Ce qui fait quasiment basculer la raison de Cereno c'est qu'une feinte image dénaturant ce qui lui est le plus cher puisse être un piège suffisant et d'autant plus efficace que la pitié de la sorte détournée est plus grande. Que la comédie des valeurs a autant de poids et d'efficacité que l'adhésion sincère à la nature, à la vérité et à la vertu, que tout système de valeurs peut être traité comme un jeu de róles : tel est le noeud du Pouvoir qui ne cesse ainsi de se jouer de la Loi. Que tout ce à quoi il a cru jusque-là ne soit plus pour Cereno, comme le fut ce qui passa pour son épée au moment des faits, "qu'un fourreau, artificiellement raidi et vide" (316), et 
qu'un être de noirceur lui ait par son action despotique révélé ce "mystère d'iniquité" : telle est l'ombre portée qui ici détruit une áme.

Mais cette ombre qui accable l'âme du personnage-Cereno au point de la faire mourir n'atteint-elle pas aussi à sa manière l'âme du narrateur et celle bien sûr du lecteur ? Ce ne sont pas seulement des considérations techniques qui peuvent justifier les "enchevêtrements" et les irrégularités du récit tel qu'il a été mené comme si le souci de coller à la vérité des faits avait exigé le passage par un gauchissement temporaire destiné à mieux faire comprendre ce qu'il s'est passé. La vérité narrative ne saurait relever ici d'une adéquation plus ou moins fiable entre les faits prétendus et leur interprétation textuelle mais elle dépend de l'homologie profonde discernable entre le noeud du Pouvoir tel que nous venons de le définir et le noeud du Récit tel que nous avons auparavant essayé de le débrouiller. Le jeu démoniaque auquel Cereno se trouve contraint, l'interprétation que ne cesse de reprendre Delano révèlent l'action occulte du pouvoirs quels qu'ils soient et la teneur de ce jeu tout comme les aléas de cette interprétation inspirent et inf́léchissent les modalités mêmes du récit. Mais il a fallu que la révélation du Mal qui rend Cereno fou d'horreur et de peur s'impose d'abord au narrateur et avec une violence analogue : elle ne doit pas non plus laisser le lecteur intact et elle va provoquer chez lui un choc spécifique. Ce Mal est celui qui naît de la facilité qu'il y a à contrefaire le vrai et le bien et de la confusion des valeurs qui en découle : il fait tomber dans un piège de portée à la fois éthique et herméneutique. Et c'est une vision du monde bien pessimiste qu'offre à l'esprit et à l'âme du lecteur le récit ainsi noué puisque celui qui vient du dehors, malgré tout ce qu'il remarque d'étrange et malgré sa sympathie, ne peut guère aider celui qui est déjà pris au piège, faute d'un point de repère stable lui permettant de décider, et que l'homme de bonne volonté est le plus facile à tromper, son bon coeur l'incitant à croire autrui quand celui-ci déplore son malheur. De plus, bien que le capitaine Delano soit "celui qui exerce la fonction cordiale seule capable de préserver de la barbarie"3, il faut à ce juste l'aide de la Providence ou du hasard pour l'emporter. Un tel récit est aussi un acte de défiance en bonne et due forme envers les capacités humaines d'interprétation car il demeure une ombre portée sur le sens produit par le texte. Que l'on songe à un événement d'origine et de nature aussi indécidables que le coup de rasoir dont est victime Babo. L'insection de l'incident dans le récit est d'abord destinée à disculper l'esclave : la cruauté gratuite est le fait du prétendu maître. Mais avec le recul induit par le renversement majeur l'on peut se demander si Babo ne s'est pas lui-même infligé cette blessure pour mieux accréditer la thèse du despotisme irresponsable de Cereno. L'on pourrait aussi penser que Cereno, se débattant

3. François George, "Angepain et Tudide au festile des Rebulites", Critique, $\mathrm{n}^{\circ} 533$, Oct. 1991, p. 789. 
vainement contre l'horreur, mais lui répondant d'une manière qui l'accentue, a bien accompli ce geste qui mêle ainsi désespoir et désir de vengeance, l'esclave ne récupérant qu'a posteriori mais avec un sens aigu de l'opportunité le fait à son profit. Point aveugle ou trou noir ? Nous sommes ici, et définitivement, dans l'incapacité de répondre à l'injonction du vieux faiseur de noeuds : "Débrouillez, tranchez, vite". Le Récit nous a pris en ses lacs qui sont aussi ceux du Pouvoir et il laisse son ombre portée sur notre coeur : notre relative impuissance herméneutique débouchant sur un sentiment d'insécurité qui peut nous pousser d'abord comme Delano à nous protéger nous-mêmes ne nous interdit toutefois pas la pitié (ce juste complément de la terreur !) :

[Delano] rabattit durement la main de Babo, mais son coeur le frappa plus durement encore. Avec une pitié infinie, il délivra Don Benito de sa propre étreinte. Ce n'était point le capitaine Delano, mais Don Benito que le noir, en sautant dans la chaloupe, avait voulu poignarder. (286) 